\title{
Research on the Early Warning Demonstration System of Freak Wave
}

\author{
Peinan Zheng ${ }^{1}$, Xiaoting $\mathrm{Li}^{2}$, Shouhua $\mathrm{Liu}^{3}$ \\ ${ }^{1} 2433$ Mailbox, Beijing 100081, China \\ ${ }^{2}$ Meteorological and oceanographic college, PLA University of Science and Technology, Nanjing 211101, China \\ ${ }^{3}$ National Marine Information Center, Tianjin 300171, China
}

\author{
畸形波预警示范系统研究设想 \\ 郑沛楠 ${ }^{1}$, 李晓婷 ${ }^{2}$, 刘首华 ${ }^{3}$ \\ ${ }^{1}$ 北京市 2433 信箱, 北京 100081 , 中国 \\ 2 解放军理工大学气象海洋学院, 南京 211101 , 中国 \\ ${ }^{3}$ 国家海洋信息中心, 天津 300171 , 中国
}

\begin{abstract}
The urgent need for ship safety and security of maritime activities, utilization of marine survey and satellite remote sensing data and other information retrieval sea waves, wave spectrum analysis of variation characteristics of freak waves occur when mixed wave conditions, research waves, surge of energy Nonlinear polymerization, explore the formation mechanism mixed wave background freak waves; based WAVEWATCH III wave model to identify closely related parameters generated factors and freak waves, study the establishment of a global early warning demonstration system freak wave, freak wave forecast exploratory prone probability and regional produce; for ocean-going ships to provide information security.
\end{abstract}

Keywords: freak waves; early warning demonstration system; research ideas

\section{摘要}

针对舰船海上活动安全保障的迫切需求, 综合利用海 洋调查数据和卫星遥感反演海面波浪等资料, 分析混 合浪条件下畸形波发生时海浪谱的变化特征, 研究风 浪、涌浪能量的非线性聚合, 探讨混合浪背景下畸形 波的生成机制; 基于 WAVEWATCH III 海浪模式, 找出与畸形波生成密切相关的参数因子, 研究建立全 球畸形波预警示范系统, 探索性预测畸形波易发生的
区域和产生的概率; 对舰船远洋航行提供信息安全保 障。

关键词: 畸形波; 预警示范系统; 研究设想

1. 引言

畸形波又称怪波, 英文名Freak wave, 通常是指海洋 中突然出现的一些怪异的巨大波浪[1]。畸形波的能 量远远超过了普通的大波浪, 可以轻易的摧毁万吨级 的轮船, 对水面舰艇航行特别是深远海航行带来极大 危害, 是海洋沉船事故的主要原因。畸形波是一种单 峰波, 与孤立波有点相似。但它的波峰很尖, 波高很 大, 这与孤立波这种推进波又有很大区别。畸形波持 续时间短, 很快消失, 也不同于驻波, 畸形波的波形 随时间变化; 与地震等因素无必然联系, 故又不同于 海啸。如图1所示为历史上拍摄到的畸形波事件。 最早的记录是由它引起1896年匍萄牙Spray号船的倾 覆。此后在欧洲北海海域、日本海域以及南非东南海 域相继出现类似的海难事故, 由于其极大的波高, 对 海上建筑物容易产生巨大危害, 因此引起人们越来越 多的重视。1965年, Draper首次提出畸形波的概念, 此外, 越来越多的学者和工程人员关注这一现象, 有 的称凶波(rogue wave)或巨波(giant wave)。近10年来, 国外对它的报道越来越多。但由于它多在未知的和不 可预料的情况下出现, 可靠的测量记录很少, 因而导 致了对其发生机理研究和定义的不完善。大多数学者 和工程技术人员从波高的角度对它进行定义, 认为波 高大于有效波高 2 倍的单波可称为畸形波。如图 2 所示 

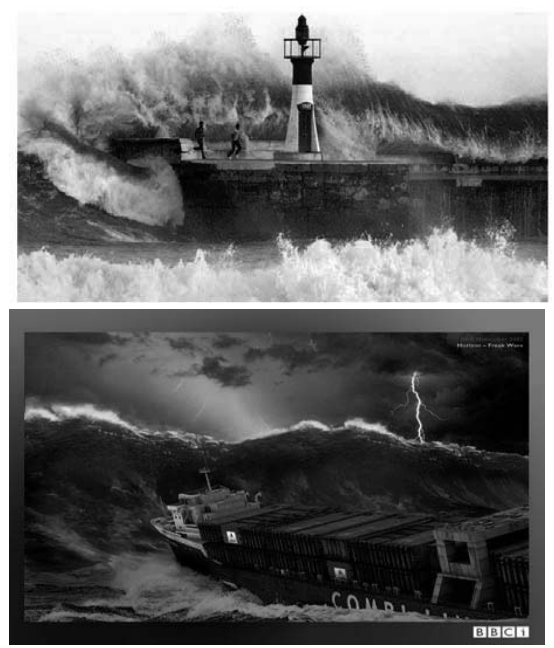

图 1 历史上的畸形波事件

为1998年2 3月在英国爱尔兰海域[2]海洋监测浮标 站记录的畸形波事件, 从图中可见, 1 个月内 $H_{M A X} / H_{1 / 3} \geq 2$ 发生了大约 50 个畸形波。

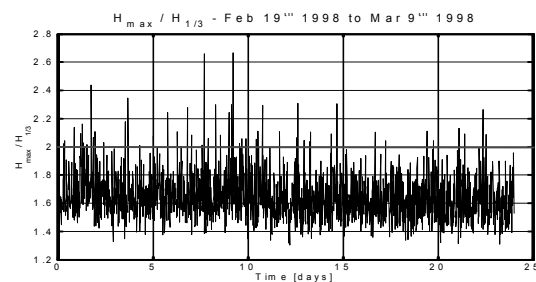

图 21998 年 2-3 月英国爱尔兰海域海洋监测浮标站记录的 畸形波事件

研究人员通过收集全球范围内的畸形波导致海 洋事故数据资料指出畸形波可能较为频发的海域有 21 处, 其中包括我国南海。1996 年台湾设立海域周 边海洋监测系统整年 4565 个波记录中获得 175 个畸 形波。2004 年欧洲航天局报道, 北海 GOMA 油田 12 年中遭遇 466 个畸形波。MAXWAVE负责人 Rosenthal 对历史数据进行分析, 得出过去 20 年中超过 200 艘 大型货轮可能因为这种异常浪的袭击而沉没。如图 3 所示为有据可查的全球畸形波事件[1]。

畸形波作为一种逐渐被人们接受的现象, 发生概 率比预计大许多。随着人类海上活动大量增加, 该现 象严重威胁人们的生命安全。它极有可能是原先所未 知的很多海难的罪魁祸首。对它的研究正在引起国际
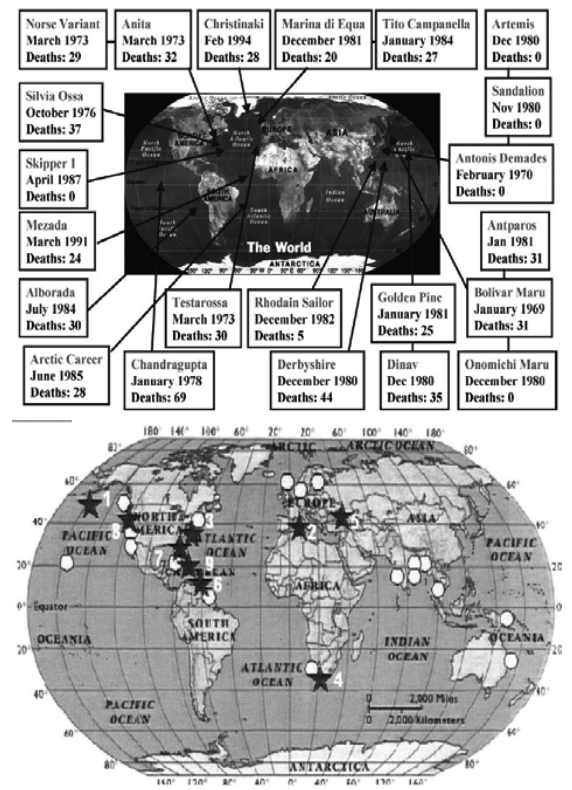

图 3 有据可查的全球畸形波事件

海洋界的关注。我国对畸形波的研究起步较晚, 研究 人员较少。虽然我国现在具有大量的海浪历史观测数 据, 但是由于数据的开放性有限, 根据它们开展的畸 形波的研究和分析工作还远远不够。

\section{2. 畸形波对远洋航行的影响}

畸形波对水面舰艇具有极大的危害和杀伤力, 随着我 国远海利益的拓展, 我国国际影响和军事活动将大量 的向深远海延伸。目前我国对包括畸形波在内的海面 风浪、涌浪及混合浪等大范围、长时序的海洋环境时 空变化规律不甚掌握, 保障手段缺乏。开展对海洋畸 形波的调查研究和建立畸形波预警示范系统, 对填补 我国畸形波的应用研究空白, 提高我国海洋环境保障 能力, 具有重要的科学意义和军事意义。

为尽快扭转对混合浪、畸形波等海洋环境保障信 息的劣势, 综合利用海洋调查数据和卫星遥感反演海 面波浪等资料, 结合数值模型, 分析混合浪条件下畸 形波发生时海浪谱的变化特征, 研究风浪、涌浪能量 的非线性聚合, 探讨混合浪背景下畸形波的生成机 制; 建立全球畸形波预警示范系统, 预测畸形波易发 生的区域和产生的概率, 为有效提高我国海洋环境保 障能力, 提供决策支持信息。 
Risk Analysis and Crisis Response in Big Data Era (RAC-16)

\section{3. 研究设想}

畸形波的调查数据较少, 对其研究和应用的进展缓 慢, 然而畸形波的对舰船海上行动安全危害巨大，必 须对其生成机制和传播规律进行掌握进而预报预警。 随着计算机技术的发展和海洋大气模型的进步, 以及 卫星遥感资料在海洋环境保障中的应用, 使得畸形波 预警示范系统的建立成为可能。因此, 综合利用海洋 调查数据和卫星遥感反演海面波浪等资料, 结合数值 模型分析混合浪条件下畸形波发生时海浪谱的变化 特征, 研究风浪、涌浪能量的非线性聚合, 探讨混合 浪背景下畸形波的生成机制; 分析 BFI 与畸形波生成 频率之间的联系，建立全球畸形波的预警系统，预测 畸形波易发生的区域和产生的概率; 研究实验室和理 论分析得出的 BFI 因子在海洋中的适用性, 验证畸形 波预警示范系统的实用性; 为我国水面舰艇远洋航行 提供信息安全保障。研究主要应解决如下几个关键性 问题:

\section{1. 畸形波生成机制研究}

畸形波波峰很尖、波高很大, 为一种单峰波, 这与孤 立波相似; 畸形波持续时间较短, 波型随时间而变化, 与驻波和地震波有很大差异。由于畸形波多发生在未 知和不可预料的情况下, 可靠的测量记录很少, 因而 导致对其发生机理研究和定义的困难。

随着大规模、长时序海洋调查数据和卫星遥感资 料的累积, 使得对畸形波的生成机制和传播规律研究 成为可能。分析观测到的畸形波发生时刻, 海浪谱的 演化特征和风浪、涌浪是否有能量聚合特征, 探讨畸 形波在混合浪背景下生成机制。

\section{2. 畸形波生成概率简易参数化}

综合利用我国大规模、长时序海浪观测数据, 进行畸 形波事件的分析和研究。通过理论分析和统计研究, 查找与畸形波生成密切相关的波浪参数因子。与畸形 波生成概率表达式的参数化进行联合分析。以海浪谱 为基础, 进行畸形波生成概率参数化的简化和数值算 法研究, 形成具有业务化运行能力的畸形波生成概率 参数化表达式。

\section{3. 全球畸形波预警示范系统研制}

在对畸形波生成机制掌握的情况下，基于 WAVEWATCH III 海浪模式[3], 找出与畸形波生成密 切相关的参数因子, 建立全球畸形波预警示范系统。 使用 Matlab 语言、Fortran 语言和 Surfer 软件、地理 信息系统 GIS, 研制全球畸形波预警示范系统可视化 软件, 实现对畸形波特征值产品的灵活查询显示, 图 形分析产品的查询汶览显示和图形即时绘制显示。

\section{4. 畸形波预警示范系统的实用性验证}

对畸形波数值数值模式输出产品进行检验。根据海洋 环境保障需求, 对模式输出海浪产品与实测数据进行 单点时间序列对比检验、与 SAR 图像[4]进行海面大 面对比检验、与前人研究结果的定性对比检验等。

\section{4. 建立畸形波预警示范系统}

\section{1. 资料的收集处理}

系统收集和整理国内外海浪业务化观测、调查资料以 及卫星遥感 SAR 图像等现场观测资料。由于现场观 测资料缺乏、来源渠道广、种类繁多、编码和格式不 一, 且有的资料质量问题严重, 因此资料预处理和质 量控制的工作量和难度很大。首先将不同来源的资料 融合在一起, 对全部资料进行格式转换、人机结合排 重, 然后根据风浪、涌浪和混合浪不同的分布特点设 计质量控制方案及人机交互检验, 为数值模式建立做 好数据准备。通过质控后的数据分析畸形波的存在和 分布情况, 研究畸形波发生时的海况特征 [7,8]。

\section{2. 寻找新的与畸形波生成密切相关的参数因子并} 进行生成概率参数化

综合利用諚备好的数据资源, 以 WAVEWATCH III 海浪模式为基础, 通过修改模式源代码, 加入独立的 BFI 输出模块并完成并行运算的调试，设计数值模拟 试验, 寻找新的与畸形波生成密切相关的参数因子。 波高的统计分布性质以波面分布特征为基础, 海浪的 非线性影响波面的统计分布特征。非线性分布特征与 正态分布的偏离程度因子 $\mathrm{C} 4$ 表达式如下。对于非线 性波浪可以用式 (2) 来表示。(2) 的计算为 6 阶积 分, 需要大量的计算时间。如何对该式进行简化是畸 形波生成概率参数化的重要一环。拟对（2）进行离 散式数值计算, 找出最核心的频率值, 最大程度减小 误差。

$$
\begin{gathered}
\mathrm{C}_{4}=\left\langle\eta^{4}\right\rangle / 3\left\langle\eta^{2}\right\rangle^{2}-1 \\
\mathrm{C}_{4}=\frac{4}{g^{2} m_{i}^{2}} \int d k_{1,2,3,4} T_{1,2,3,4} \delta_{1+2-3-4}\left(\omega_{1} \omega_{2} \omega_{3} \omega_{4}\right)^{1 / 2} \frac{N_{1} N_{2} N_{3}}{\Delta \omega} \\
\Delta \omega=\omega_{1}+\omega_{2}-\omega_{3}-\omega_{4}
\end{gathered}
$$

\section{3. 全球畸形波预警示范系统研制}

全球畸形波预警示范系统的主要理论依据是, 目前理 论研究和实验室模拟发现: 在窄谱条件和波浪方向展 角非常小的情况下, 畸形波的生成频率和 BFI 参数因 子有极大的相关性。Janssen 和 Mori (2006) 给出了 
Risk Analysis and Crisis Response in Big Data Era (RAC-16)

这种条件下的畸形波的生成概率表达式[6]。BFI 和概 率表达式如下。

$$
\begin{aligned}
& B F I=k_{o} m_{o}^{1 / 2} Q_{p} \sqrt{2 \pi} \\
& Q_{P}=2 m_{0}^{-2} \int_{0}^{\infty} d \sigma \sigma\left[\int_{0}^{2 \pi} F(\sigma, \theta) d \theta\right]^{2} \\
& \mathrm{P}_{\text {freak }}=1-\exp \left[-\beta N\left(1+8 \kappa_{40}\right)\right] \\
& \kappa_{40}=\frac{\pi}{\sqrt{3}} B F I^{2}
\end{aligned}
$$

其中 $F(\sigma, \theta)$ 为一维海浪谱, $\beta$ 为常数 $\mathrm{e}^{-8}, \mathrm{P}_{\text {frak }}$ 为畸开後皮的发生概率

以 WAVEWATCH III 海浪模式为基础, 进行数值预报 试验, 输出网格点的二维海浪谱, 用辛普生算法 [9] 对二维海浪谱进行数值积分, 得出网格点的 BFI 和畸 形波发生概率。从 BFI 和畸形波发生概率即可判断某 一海域的畸形波发生情况, 从而提供为海上舰艇活动 提供预报预警。全球畸形波预警示范系统分辨率不低 于 $1^{\circ} \times 1^{\circ}$; 预报时效为 7 天逐 6 小时预报; 波浪 谱高频不小于 $5 \mathrm{~Hz}$, 低频不大于 $0.01 \mathrm{~Hz}$, 角度分辨率 不低于 $10^{\circ}$ 。如图 4 所示为全球畸形波预警示范系 统流程图。

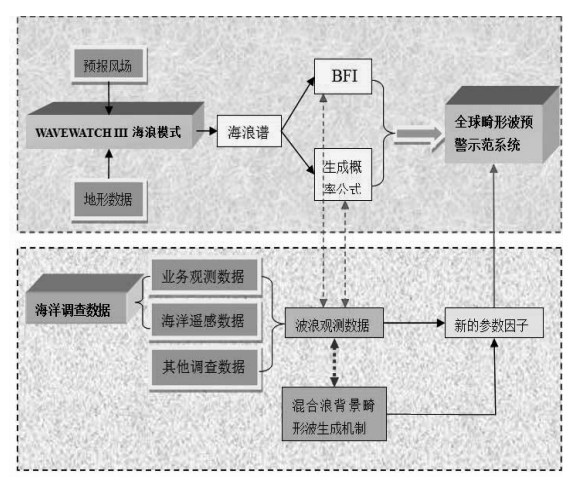

图 4 全球畸形波预警示范系统流程图

\section{4. 畸形波预警示范系统的实用性验证}

对畸形波数值模式输出产品进行检验验证。根据海洋 环境预报保障需求, 对模式输出的海浪产品 (包括风 浪、涌浪、混合浪以及畸形波) 与实测数据进行单点 时间序列对比检验、与 SAR 图像进行海面大面对比 检验、与前人研究结果的定性对比检验等 [10]。

\section{5. 结论与讨论}

本文主要论述了如何建立畸形波预警示范系统 的研究设想, 概括来说主要分为如下几个步骤。首先
完成我国海浪观测历史数据的分析和处理, 给出我国 畸形波的数量和分布特点; 然后探寻畸形波生成和传 播规律, 给出参数化的畸形波生成概率表达式; 其次 将参数化畸形波生成概率表达式嵌入到第三代海浪 模式 WAVEWATCH III 中, 建立全球海浪数值预报模 型, 制作海浪数值预报产品（波高、波向、周期、海 浪谱等常规海浪预报产品以及 BFI、畸形波生成概 率、风浪涌浪能量比、波浪破碎率等海浪综合应用产 品); 最后建立全球畸形波预警示范系统, 实现对畸 形波的灵活查询显示和图形即时绘制显示, 以及畸形 波生成概率、分布状况和传播规律研究成果的灵活查 询显示。

\section{参考文献}

[1] 杨冠声, 董艳秋, 陈学间. 畸形波. 海洋工程, 120(14):105-108, 2002.

[2] Havers, S, O J Andersen. Freak Waves: Rare Realizations of a Typical Population or Typical Realizations of a Rare Population. ISOPE, pp.1036-1039, 2003.

[3] Bi F., Song J. Wu K.J. Evaluation of the simulation capability of the Wavewatch III model for Pacific Ocean wave. Acta Oceanol. Stn., 34(9): 43-57, 2015.

[4] Dankert, H, J Horstman. Detection of Waves Groups in SAR Images and Radar-Image Sequences. TGRS, pp.22-32, 2013.

[5] 黄国兴. 畸形波的模拟方法及基本特性研究. 大 连理工大学博士学位论文, 2002.

[6] 陶爱峰. 深水波列演变及畸形波生成机制之数 值探讨. 河海大学博士学位论文, 2007.

[7] 伍进, 肖辉. 浅谈畸形波的波形模拟. 中国海洋 平台, 20(4): 1-9, 2015.

[8] Wang W D, S Qiao, F L Zeng, G Jun. An Approach to Ranking Integrated Models for Risk Assessment by Using the Internet of Intelligences. Journal of Risk Analysis and Crisis Response, 6(1): 2-9, 2016.

[9] Kristen A S, L A. Jordan. BP's Reputation Repair Strategies during the Gulf Oil Spill. Journal of Risk Analysis and Crisis Response, 5(1): 2-15, 2015.

[10] Sviatoslav T. Average Life Expectancy as a Criterion for Regional Risk Management. Journal of Risk Analysis and Crisis Response, 4(1): 10-19, 2014. 